\title{
Lorsque la répression est un plaisir : le militantisme au Parti National Bolchévique russe
}

When Repression is a Pleasure: Activism at the Russian National Bolshevik Party

\section{Véra Nikolski}

\section{OpenEdition}

12 Journals

\section{Édition électronique}

URL : http://journals.openedition.org/conflits/18684

DOI : $10.4000 /$ conflits. 18684

ISSN : $1777-5345$

Éditeur :

CECLS - Centre d'études sur les conflits - Liberté et sécurité, L'Harmattan

\section{Édition imprimée}

Date de publication : 15 juin 2013

Pagination : 13-28

ISBN : 978-2-343-01116-5

ISSN : 1157-996X

\section{Référence électronique}

Véra Nikolski, « Lorsque la répression est un plaisir : le militantisme au Parti National Bolchévique russe », Cultures \& Conflits [En ligne], 89 | printemps 2013, mis en ligne le 15 juin 2014, consulté le 31 mars 2021. URL : http://journals.openedition.org/conflits/18684; DOI : https://doi.org/10.4000/ conflits. 18684 


\section{Lorsque la répression est un plaisir : le militantisme au Parti National Bolchévique russe}

\section{Véra NIKOLSKI}

Véra Nikolski est sociologue et politiste, docteure en science politique et chercheure associée an Centre européen de sociologie et de science politique (CESSP-Paris). Ses travaux portent sur le champ politique russe et le militantisme radical. Un ouvrage issu de sa thèse de doctorat - National-bolchevisme et néo-eurasisme dans la Russie contemporaine : la carrière militante d'une idéologie - est actuellement sous presse aux éditions MareEMartin.

I e 14 décembre 2004, le fonctionnement routinier du parloir de l'adminisLtration présidentielle de la Fédération de Russie, situé non loin du Kremlin, a été perturbé par un événement exceptionnel : un groupe d'une quarantaine de jeunes gens a fait irruption dans le bâtiment pour exiger une rencontre avec le Président, puis, devant le refus du personnel, a occupé l'un des bureaux du parloir pour s'y barricader. L'occupation a duré trente-cinq minutes, temps nécessaire aux forces de l'ordre pour arriver sur les lieux, débloquer la porte et arrêter les jeunes gens. Ces derniers appartenaient tous à une organisation militante connue sous le nom du Parti national bolchevique (NBP) ${ }^{1}$; le déroulement de l'événement ainsi que son dénouement - l'arrestation des participants - ressemblaient à ceux de plusieurs autres « actions directes 2 » spectaculaires conduites par les membres de cette organisation depuis 1998.

1. Nacional-bol'ševistskaâ partiâ. L'organisation a été officiellement interdite pour " extrémisme » en 2007. En dépit de sa non-existence légale depuis cette date, nous utiliserons le sigle NBP par commodité, même lorsqu'il s'agira d'événements postérieurs à 2007. Nous emploierons également le terme indigène " parti », bien que ce statut n’ait jamais été accordé légalement à l'organisation.

2. Le terme d'" actions directes " ("akcii prâmogo dejstviâa », littéralement "actions d'action directe »), importé et revendiqué par le NBP, désigne un mode d'action protestataire exploité dès le début des années 1980 par les mouvements écologistes et altermondialistes des pays occidentaux, qui consiste à mettre en scène des coups d'éclat censés attirer l'attention médiatique sur un enjeu spécifique. 
Apparue au lendemain de l'effondrement de l'URSS, au début des années 1990, sous l'impulsion de l'écrivain bohème Édouard Limonov et du penseur nationaliste autodidacte Alexandre Dugin, l'organisation se présente d'emblée comme une entreprise hétérodoxe ${ }^{3}$. En effet, elle allie une idéologie syncrétique d'extrême-gauche et d'extrême-droite, et un style délibérément provocateur, attirant principalement les jeunes (entre seize et vingt-cinq ans) de milieux modestes mais relativement cultivés, notamment grâce à son interpénétration avec le monde de la contre-culture underground ${ }^{4}$. Tout d'abord spécialisée dans les activités de réflexion et les happenings avant-gardistes, l'organisation commence à partir de la fin des années 1990 à mener des actions plus directement politiques - meetings et manifestations sous des slogans extrêmes, puis «actions directes ». Ultranationaliste par ses prises de position lors de sa création, l'organisation évolue dans les années 2000 vers une alliance avec l'opposition démocratique et libérale 5 .

Le parcours de cette organisation en fait un terrain propice pour appréhender la relation entre le militantisme et la répression dont elle est l'objet. Depuis l'adoption de répertoires d'action spectaculaires comme les « actions directes »- et, plus tardivement, les « marches du désaccord » conjointes avec l'opposition libérale - l'organisation est confrontée à une répression constante de la part des forces de l'ordre (police, OMON ${ }^{6}$, forces du FSB 7 ). Surveillés au quotidien, ses militants sont régulièrement arrêtés à l'issue des meetings, voire avant, et fréquemment molestés. Les locaux sont saisis (l'organisation a eu trois QG successifs). Enfin, elle est interdite pour « extrémisme » en 20078 et survit ensuite sur un mode semi-clandestin ; mais ses effectifs déclinent, passant de plusieurs milliers de militants à une centaine entre le début et la fin des années 2000.

3. Voir à ce propos Nikolski V., "Le Parti National Bolchevique russe : une entreprise politique hétérodoxe ", Critique Internationale, ${ }^{\circ}$ 55, 2012, pp. 93-115.

4. Sur les liens entre le NBP et la contre-culture, notamment musicale, voir Mathyl M., « Nationalisme et contre-culture jeune » in Laruelle M. (ed.), Le Ronge et le noir. Extrême droite et nationalisme en Russie, Paris, CNRS Éditions, 2007, pp. 115-137.

5. Dans le champ politique russe, ces termes renvoient aux forces politiques qui saluent l'effondrement de l'URSS, s'opposent au communisme et au nationalisme et revendiquent un attachement au modèle occidental, fondé sur la démocratie libérale, l'économie de marché et un État de droit. Associées aux réformes de Boris Eltsine, elles ne rencontrent que peu de soutien auprès de la population durant les années 1990 et 2000 . Pour plus de détails sur la structure du champ politique post-soviétique et sur la place qu'y occupe le NBP, consulter Nikolski V., National-bolchevisme et néo-eurasisme dans la Russie contemporaine : la carrière militante d'une idéologie, à paraître aux éditions Mare\&Martin.

6. Otrâd milicii osobogo naznačeniâa ("Brigade de police spéciale») est une unité de police spécialisée dans le maintien et le rétablissement de l'ordre dans le cadre urbain, y compris à travers l'usage de la force (notamment lors des manifestations), équivalent des CRS françaises.

7. Federal'nầ služba bezopasnosti ("Service fédéral de sécurité ») désigne les services secrets intérieurs de la Russie, héritiers du KGB.

8. Loi fédérale «Sur l'opposition à l'extrémisme » (O protivodejstvii èkstremizmu) du 18 février 2005. 
La relation entre militantisme et répression est, dans le cas du NBP, représentative de l'ambivalence que la littérature sociologique n'a cessé de souligner. Combes et Fillieule rappellent que pour les tenants de la théorie de la frustration, la répression aurait un effet positif sur la mobilisation, en radicalisant les contestataires. À l'inverse, dans le cadre du paradigme de la mobilisation des ressources où l'on raisonne en termes de coûts/avantages, la répression aurait un effet dissuasif, pouvant « décapiter un mouvement, freiner les velléités activistes et mettre un terme à la protestation ${ }^{9} »$. Si aucune relation univoque ne peut ainsi être observée, les auteurs rappellent que les recherches les plus fructueuses - notamment celles de Opp et Roehl 10 - « invitent à formuler la question autrement, de manière à discerner quels effets sont observés (spécification des relations) et sous quelles conditions (contextualisation des relations) 11 ». Analyser les effets différenciés de la répression implique alors de tenir compte de facteurs tels que la nature de la répression, l'échelle temporelle de ses effets ou encore l'aspect objectif ou subjectif du risque encouru.

C'est une perspective similaire que nous adopterons ici, en nous intéressant pourtant à des facteurs d'ordre différent. Pour comprendre la logique des effets de la répression sur une organisation, il nous semble indispensable, d'une part, de considérer la structure des rétributions de l'organisation, ellemême fonction de la place de cette dernière dans le champ politique, et d'autre part, d'examiner le contexte sociopolitique global dans lequel l'interaction entre l'organisation et le pouvoir prend place. En effet, la prise en compte de ces éléments permet d'apprécier la rentabilité de la répression pour une organisation particulière et dans un contexte donné, cette rentabilité étant fonction des caractéristiques de l'organisation et de celles de son environnement.

Nous appliquerons donc ce programme méthodologique au NBP, en nous intéressant, dans un premier temps, aux facteurs rendant la répression rentable pour l'organisation à une certaine étape de sa trajectoire, tant par rapport à l'extérieur (en termes de stratégie d'image) que par rapport à l'intérieur (en termes de mobilisation des militants) ; puis nous montrerons que cette rentabilité a trait à la nature particulière des rétributions circulant dans cette organisation marginale et atypique, rétributions qui exigent une série de conditions pour pouvoir être distribuées ${ }^{12}$.

9. Combes H., Fillieule O., «De la répression considérée dans ses rapports à l'activité protestataire. Modèles structuraux et interactions stratégiques ", Revue française de science politique, vol. 61, 2011, pp. 1047-1072.

10. Opp K. D., Roehl W., "Repression, Micromobilization, and Political Protest”, Social Forces, 69 (2), 1990, pp. 521-547.

11. Combes H., Fillieule O., op. cit., p. 1052.

12. Cet article reprend une partie de l'argumentation développée dans notre thèse de doctorat (Nikolski V., Le moment escapiste. Militantisme et production théorique dans une conjoncture de crise. Deux mouvements de jeunesse radicaux (NBP et ESM) dans la Russie contemporaine, Paris 1,2010), qui s'appuie sur une enquête de terrain de type ethnographique effectuée au sein du NBP moscovite entre 2005 et 2008, avec quelques incursions au sein de la cellule saint-pétersbourgeoise. Les sections de province - au demeurant moins nombreuses et aux 


\section{Le rendement stratégique de la répression}

Le courant de pensée qui s'est attaché à démontrer la variété des modalités d'action des entreprises contestataires - le paradigme de mobilisation des ressources - a souligné que le recours, par un mouvement, à une forme d'action particulière ne se fait pas au hasard, mais dépend de la structure d'opportunités qu'offre le contexte, et notamment de l'attitude des autorités ${ }^{13}$. Les moyens retenus au sein $\mathrm{du}$ " répertoire d'action collective » existant reflètent ainsi l'adaptation des acteurs aux contraintes extérieures.

Dans cette conception clairement interactionniste du choix des modes d'action ${ }^{14}$, le statut de la répression peut pourtant être au moins double. Au premier abord, elle peut être vue uniquement comme un facteur qui élève le coût d'un mode d'action particulier ; dans certains de ses travaux, Charles Tilly a ainsi insisté sur cet effet dissuasif de la répression, l'augmentation des coûts de l'engagement pouvant conduire à l'affaiblissement, voire à l'arrêt de la mobilisation ${ }^{15}$. Pourtant, et tout en restant dans un raisonnement de type stratégique, la répression peut au contraire devenir un atout pour une organisation, élevant non seulement le coût, mais également le rendement d'un moyen d'action particulier, notamment par la résonnance que son utilisation peut avoir dans les médias, et donc par le surplus de notoriété, et éventuellement de compassion, qu'elle peut générer dans l'opinion publique.

Les exemples d'une telle utilité stratégique de la répression sont nombreux : on peut ainsi évoquer certains cas de désobéissance civile, comme les faucheurs d'OGM en France, mais également celui du PCF des années 1950 étudié par Vanessa Codaccioni. S’inspirant du cadre de pensée gofmanien ${ }^{16}$, elle analyse en effet les «interactions répressives » entre les opposants communistes et l'État comme la résultante d'une offre et d'une demande de répressions. Considérant que "l'émergence de certaines affaires communistes est non seulement envisagée et souhaitée par certains membres du groupe dirigeant mais réellement "programmée” par eux à la manière de véritables “scénarios" 17 », elle entreprend ainsi de saisir « quand, comment et pourquoi une

actions moins médiatisées - restent en dehors du cadre de l'étude, tout comme la période postérieure à l'élection de Dmitri Medvedev. Outre l'observation régulière, vingt-quatre entretiens avec les militants de l'organisation ont été réalisés, six entretiens avec d'ex-militants, ainsi que des entretiens avec les pères fondateurs de l'organisation - Alexandre Dugin et Edouard Limonov - et plusieurs de leurs alliés et compagnons de route de l'époque. Pour plus de détails sur la démarche méthodologique, voir Nikolski V., « La valeur heuristique de l'empathie dans l'étude des engagements “répugnants” ", Genèses, n 84, 2011, pp. 113-126.

13. Voir en particulier Tilly C., La France conteste, de 1600 à nos jours, Paris, Fayard, 1986.

14. On peut trouver chez Michel Offerlé la même conception interactionniste du "répertoire d'action ", voir Offerlé M., "Retour critique sur les répertoires d'action collective (XVIIIXXIe siècles) », Politix, $n^{\circ} 81,2008$, pp. 181-202.

15. Tilly C., From Mobilization to Revolution, Reading, Mass., Addison-Wesley, 1978.

16. Goffman E., Les cadres de l'expérience, Paris, Minuit, 1991.

17. Codaccioni V., Punir les opposants. Une sociologie historique des "procès politiques » en temps de crise. Les interactions répressives entre le PCF et l'Etat (1947-1962), Paris 1, 2011, p. 154. 
institution politique comme le PCF décide d'utiliser la violence et la répression à des fins politiques et d'encourager l'arrestation ou l'emprisonnement de ses membres 18 ».

Sous un angle similaire, on peut analyser les interactions répressives entre le pouvoir russe et le NBP dans les années 2000. En effet, la répression est, dans ce cas, le résultat d'un échange de heurts entre l'organisation et les agents du maintien de l'ordre. Comme le montrent les entretiens avec les militants de la première heure et les fondateurs de l'organisation, la répression - arrestation, procès et emprisonnement des militants - est ainsi une conséquence directe de l'adoption, par le NBP, des actions directes spectaculaires dont les militants savent pertinemment qu'elles vont produire cet effet. Cette répression sciemment provoquée devient un pilier central de la communication de l'organisation, que nous avons repéré dès les premiers pas sur le terrain, par le biais de la publicité faite autour de ceux que l'organisation qualifie de «prisonniers politiques », bien qu'ils soient légalement condamnés pour hooliganisme ou troubles à l'ordre public.

Si de nombreux procès aboutissent à des condamnations légères (avec sursis, ou bien aux classiques quinze jours de détention pour « hooliganisme ${ }^{19}$ »), certaines sont bien plus lourdes. Ainsi en est-il des deux actions sans doute les plus connues des militants du NBP : celle de l'occupation du bureau du ministre de la santé et du développement social Mikhail Zurabov, le 2 août 2004 (action dirigée contre la loi sur la monétisation des avantages sociaux ${ }^{20}$ ), et celle, déjà évoquée, de l'occupation du parloir de l'administration présidentielle de la Fédération de Russie, le 14 décembre 2004. Sept des participants à la première sont condamnés à purger entre deux ans et demi et cinq ans de prison ferme ; quant aux auteurs de la seconde, au nombre de trente-neuf, leur cas est aggravé en raison de l'importance de l'objet auquel ils s'en sont pris. Accusés de tentative de prise illégale du pouvoir, ils risquent vingt ans de prison ferme. L'affaire des jeunes « décembristes »-comme la presse et les militants eux-mêmes désignent les natsbols 21 jugés - fait grand bruit dans les médias, les comparaisons avec les procès staliniens allant bon train dans ceux de l'opposition. La charge ayant finalement été requalifiée en participation aux troubles à l'ordre public ${ }^{22}$, trente et un militants sont condamnés à des peines avec sursis, et seuls huit à des peines de prison ferme, allant d'un an et demi à trois ans et demi.

18. Ibid., p. 106.

19. Article 20.1 du Code Administratif de la Fédération de Russie.

20. Loi fédérale du 22 août 2004, N 122-FZ.

21. Contraction des mots «national-bolchevique» (nacional-bol'ševik), auto-désignation des militants du NBP reprise dans les médias et le langage courant.

22. Article 212 du Code Pénal de la Fédération de Russie. 
Ces procès, les incarcérations auxquelles ils donnent lieu et la communication que le NBP développe autour de ses « prisonniers politiques » font au moins autant pour la publicité du parti que les actions directes elles-mêmes. Les visages des jeunes militants incarcérés sont ainsi à l'honneur non seulement sur le site Internet de l'organisation, mais également sur les affiches exhibées lors des meetings. Leurs noms sont repris dans les publications et interviews des dirigeants, et notamment celles de Limonov dont la notoriété d'écrivain donne accès à un auditoire non négligeable. Cette publicité explique que les effectifs du parti croissent particulièrement pendant la première moitié des années 2000, riche en actions directes, procès et emprisonnements. Le petit collectif underground des années 1990 devient alors une organisation politique à portée nationale, forte de plusieurs milliers de militants actifs.

Le séjour en prison du leader du parti lui-même fait également beaucoup de bruit. Arrêté en avril 2001 en Altaï avec un groupe de jeunes natsbols, Limonov est soupçonné de préparer un coup d'État paramilitaire visant l'occupation d'une partie du Kazakhstan et accusé de détention illégale d'armes, de tentative de création de groupements armés, de préparation d'actes terroristes et d'appels à renverser l'ordre constitutionnel. Après un long procès, Limonov est condamné à quatre ans de prison ferme, mais libéré avant terme, le 30 juin 2003. L'incarcération du leader n'a pas porté atteinte au parti ; elle contribue au contraire fortement à la visibilité de la structure NBP, les effectifs continuant de croître en son absence.

La répression génère un autre bénéfice stratégique pour l'organisation : elle lui permet de transformer sa structure d'alliances à un moment où les thématiques nationalistes, créneau privilégié de l'opposition durant les années 1990, changent de camp depuis l'avènement au pouvoir de Vladimir Poutine. Avec l'orientation patriotique de ce dernier, le camp libéral et démocratique peu soutenu par l'électorat car associé aux réformes libérales impopulaires du début des années 199023 - porte désormais le discours le plus radicalement opposé à celui du pouvoir, alors que l'opposition nationaliste et communiste - très vigoureuse durant les années Eltsine - arrive bien plus difficilement à formuler des doléances à l'encontre d'un leader qui semble reprendre une partie de son propre argumentaire. Le contexte international - révolutions « de couleur » dans les pays voisins - paraît au même moment donner une nouvelle chance aux contestations d'inspiration démocratique. Le NBP pouvant fournir des troupes de militants aguerris et habitués aux actions de rue, et les libéraux-démocrates assurer la crédibilité, mais également le financement du mouvement, l'alliance peut être mutuellement profitable. Elle n'enthousiasme certes guère les militants national-bolcheviques du rang, qui restent majoritairement hostiles aux idées libérales; mais comme nous le verrons plus loin,

23. En décembre 2003, aucun des partis démocratiques et libéraux n’a réussi à franchir la barre des $5 \%$ des voix nécessaires pour accéder à la Douma. 
l'idéologie compte moins pour eux que les possibilités d'action, et le succès de ces dernières semble alors dépendre de la nouvelle stratégie adoptée.

Dans ce contexte, le programme ultranationaliste et impérialiste du NBP des années 1990 est radicalement modifié en 2004 autour de l'opposition à l'autoritarisme de Poutine et de la défense des droits de l'homme, permettant au NBP de fonder en logique la naturalité de son rapprochement avec les libéraux. Davantage que son programme, ce sont les poursuites dont le NBP devient progressivement l'objet, et l'existence de « prisonniers politiques » qui lui permettent de trouver une base d'entente avec l'opposition démocratique, en réactivant notamment l'imaginaire de la dissidence de l'époque soviétique, chère à cette dernière.

Si toutes les tentatives de rapprochement entreprises à partir de 2004 n'aboutissent pas ${ }^{24}$, le NBP finit par s'intégrer dans la coalition l'Autre Russie qui regroupe un ensemble de mouvements et d'acteurs politiques démocrates et libéraux : on y retrouve ainsi le joueur d'échecs Garri Kasparov (et son mouvement OGF, «Front civique uni »), l'ancien Premier ministre Mikhail Kasianov (leader du mouvement RNDS, «Union populaire-démocratique de Russie ») 25, mais également les mouvements de jeunesse Oborona ("Défense ») et Smena ( Relève »), ainsi que le Mouvement pour la défense des droits de l'homme, Limonov et Kasparov devenant les principaux dirigeants de la structure. Le NBP est alors financé à travers l'OGF, et les réunions du NBP moscovite se tiennent au local de ce mouvement, avant que la coalition ne se disloque en 2010.

\section{L'héroïsme comme valeur et comme plaisir}

L'intérêt de la répression pour le NBP ne se résume pourtant pas aux seuls gains en termes d'image, de notoriété ou de tactique d'alliances; plus encore que cette rentabilité externe, c'est la rentabilité interne de la répression qui explique l'offre de l'organisation. Comme nous l'avons vu, les travaux sur les dynamiques des mobilisations collectives ont depuis longtemps souligné que la répression peut parfois amplifier l'action collective plutôt que la freiner. Le cas du NBP est, en l'occurrence, très similaire à celui des mouvements de la Nouvelle gauche aux États-Unis et au Japon étudiés par Gilda Zwerman et Patricia Steinhoff. En effet, les auteurs montrent que le déclenchement de la répression étatique a globalement un effet négatif sur le mouvement, conformément au paradigme de l'acteur rationnel : l'élévation du coût de l'engagement conduit la plupart des militants à se retirer ou à choisir des formes plus modérées de contestation; en revanche, une minorité de convaincus persévère

24. Ainsi, l'alliance ne se fera ni avec le parti Iabloko de Grigori Iavlinski, ni avec le SPS (Union des Forces de Droite).

25. Kasianov quitte la coalition dès 2007. 
et se radicalise, répondant à la violence par la violence, comme si la répression stimulait l'intensité de l'engagement ${ }^{26}$. «Pour ces insurgés, la répression représentait quelque chose d'autre qu'un obstacle à la contestation, ou une raison de fuir » : elle constituait pour eux « une opportunité d'intensifier leur lutte », notent ainsi les auteurs ${ }^{27}$.

Cette nouvelle phase de radicalisation de la Nouvelle gauche portée par une minorité très engagée représente, pour Zwerman et Steinhoff, un fait distinct de la mobilisation plus conventionnelle qui la précède. Les auteurs proposent de parler d'une "mobilisation de résistance 28 ", phénomène que l'on rencontre dans de nombreuses configurations répressives. Pour l'expliquer, les facteurs habituellement mis en avant font appel soit aux dynamiques émotionnelles provoquées par la répression, la colère et le sentiment d'injustice amplifiant la mobilisation ${ }^{29}$ - thèse qui profite du renouveau de l'attention aux émotions dans l'analyse de l'action collective 30 - soit aux incitations sélectives que les individus peuvent trouver dans la participation à la mobilisation dans un contexte répressif 31 . Dans le cas du NBP où la répression produit bien un effet d'intensification qualitative (sinon d'amplification quantitative) de l'engagement, l'explication exige d'emprunter aux deux schémas d'analyse, pour montrer que ce sont précisément les émotions que la répression produit qui peuvent être considérées comme des incitations sélectives immatérielles.

Nous partons de l'idée déjà ancienne d'Albert Hirschman - récemment rappelée par Frédéric Sawicki et Johanna Siméant 32 - qui remarque, dans Bonbeur privé, action publique, que militer est toujours, dans une certaine mesure, un plaisir en soi, comme l'est le pèlerinage pour un homme religieux, pour lequel « le segment de plaisir pénètre le segment de coût et l'imprègne de sa propre expérience 33 ». La nature précise de ce plaisir en soi retiré du mili-

26. Zwerman G., Steinhoff P., "When activists ask for trouble: State-dissident interactions and the New-Left cycle of resistance in the United Stated and Japan”, in Davenport Ch., Johnston H., Mueller C., Repression and mobilization, Minneapolis, University of Minnesota Press, 2005, pp. 85-107.

27. Ibid., p. 88 (traduction personnelle).

28. "Mobilization of resistance " (Idem).

29. Parmi les nombreux exemples, McAdam D., Tarrow S., Tilly C., Dynamics of Contention, Cambridge, Cambridge University Press, 2001 et Brockett C., Political Movements and Violence in Central America, New York, Cambridge University Press, 2005.

30. Goodwin J, Jasper J. M., Polletta F., Passionate Politics: Emotions and Social Movements, Chicago, University of Chicago Press, 2001 ; Goodwin J., Jasper J. M. (ed.), Rethinking Social Movements: Structure, Meaning, and Emotion, Lanham (Md.), Rowman and Littlefield, 2004 ; Traïni C. (ed.), S'émouvoir pour la cause. Sensibilisation et mobilisations collectives, Paris, Presses de Science Po, 2008 ; Traïni C. (ed.), Émotions... Mobilisation!, Paris, Presses de la FNSP, 2009.

31. Opp K. D., "Repression and Revolutionary Action: East Germany in 1989”, Rationality E Society, vol. 6, 1994, pp. 101-138 ; Moore W. H., "Rational Rebels: Overcoming the FreeRider Problem”, Political Research Quarterly, n 48, 1995, pp. 417-454.

32. Sawicki F., Siméant J., «Décloisonner la sociologie de l'engagement militant. Note critique sur quelques tendances récentes des travaux français ", Sociologie du travail, 2009, n 51, pp. 97-125. 
tantisme - qui peut, puisqu'il rémunère le militant, être considéré comme une «rétribution » au sens de Daniel Gaxie ${ }^{34}$ - n’est pourtant pas précisée chez Hirschman, alors qu'en considérant des partis ou mouvements concrets, on ne peut que constater que des groupes de nature différente génèrent des plaisirs eux aussi différents ${ }^{35}$. En effet, outre les plaisirs en soi correspondant aux rétributions classiques du militantisme énumérées dans l'article fondateur de Gaxie (comme le plaisir de la camaraderie), qui sont généralement présents, à des degrés divers, dans toute formation à vocation politique, il peut y avoir des plaisirs en soi plus spécifiques. Et - c'est l'hypothèse ici défendue - le plaisir spécifique de militer au NBP a partie liée avec la répression dont l'organisation est l'objet.

En analysant le mode d'engagement des natsbols, nous avons choisi comme point de départ des indicateurs objectifs - lieux, pratiques, façon de parler ou de se présenter - qui singularisent leur groupe, pour mettre en exergue le sens qu'ils ont aux yeux des militants. Or, la systématisation des éléments empiriques, nécessairement épars et hétérogènes, issus de l'observation et des entretiens, permet de mettre en évidence un certain ethos ${ }^{36} \mathrm{du}$ militant de l'organisation dont la principale manifestation extérieure est la mise en scène systématique de l'héroïsme individuel et collectif. Transposable, ce principe directeur ne se révèle pas uniquement dans le répertoire d'action spectaculaire pratiqué par l'organisation, mais ordonne bien plus largement l'ensemble des façades repérables dans les interactions, se manifestant à la fois dans le comportement des acteurs et dans les décors dont ils s'entourent dans leur activité militante ${ }^{37}$.

La mise en scène de l'héroïsme passe, pour commencer, par le vocabulaire des natsbols renvoyant au champ sémantique de la guerre : les enquêtés se désignent ainsi comme des « combattants », des « guerriers », des "frères », des « héros », et se réfèrent aux valeurs telles que l' « honneur », la « vengeance », le « courage », le « sacrifice » ou la « lutte». Elle se manifeste égale-

33. Hirschman A. O., Bonbeur privé, action publique, Paris, Fayard, 1983, p. 152 (en italique dans le texte).

34. Gaxie D., "Économie des partis et rétributions du militantisme », Revue française de science politique, vol. 27, n 1, 1977, pp. 123-154 ; Gaxie D., « Rétributions du militantisme et paradoxes de l'action collective ", Revue suisse de science politique, vol. 11, 2005, pp. 157-188.

35. Nous faisons ainsi nôtre la remarque de Michel Offerlé (critiquant la vision étroitement utilitariste de la notion d' « intérêt » qui ramène les motivations que les individus peuvent avoir à la participation à l'action politique aux seules rétributions stricto sensu intéressées) selon laquelle « [1]'important (autre définition de l'intérêt) n'est jamais identique, mais toujours spécifique » (Offerlé M., Les Partis politiques, Paris, PUF, 2006 [1987], p. 72).

36. L'importance, dans cet ethos, de la dimension émotionnelle fait que l'on pourrait également y appliquer le terme d' " habitus émotionnel » (emotional habitus) proposé par Anne Kane (voir Calhoun C., "Putting Emotions to Their Place", in Goodwin J., Jasper J. M., Polletta F. (eds.), Passionate Politics: Emotions and Social Movements, Chicago, University of Chicago Press, 2001, pp. 45-57).

37. On se réfère ici au cadre conceptuel goffmanien (Goffman E., La présentation de soi. La mise en scène de la vie quotidienne I, Paris, Minuit, 1973). 
ment dans l'organisation des espaces communs dans lesquels ils évoluent - un local du parti organisé comme une forteresse et appelé «Bunker », des squats communautaires où logent les militants, qui se distinguent par leur austérité ostentatoire - et dans la présentation de soi des membres de l'organisation : fréquence d'habits paramilitaires, défilés belliqueux, mais également de nombreux rituels de la vie quotidienne censés en permanence rappeler le danger d'être suivis, écoutés ou arrêtés par les forces de l'ordre (brouillage des pistes lors des déplacements en ville, geste d'éteindre, voire d'éloigner les portables pour les conversations importantes, réflexe d'aller dormir chez un ami en cas de soupçon d'arrestation, etc.). Le culte des « prisonniers politiques » s'inscrit dans la même logique. Sans cesse magnifiés, les détenus sont érigés en exemples à suivre, véritables martyrs indigènes.

Si ces éléments doivent leur existence à la répression bien réelle dont ces militants font l'objet, leur mise en avant systématique dans les interactions, qui confine à l'exhibition volontaire, montre que le danger et l'inconfort ne sont pas simplement supportés par les militants en tant que coûts de leur pratique. Au contraire, l'héroïsme, valeur centrale de l'organisation, permet de classer les militants sur une échelle de prestige interne, générant l'émotion positive de fierté chez ceux qui s'exposent le plus au danger : la fierté d'être des braves et celle d'être les ennemis désignés de l'État (et donc ses égaux).

Or, dans les deux cas, c'est bien la répression qui joue le rôle de preuve ou de pierre de touche de l'hérö̈sme des militants : d'une part, la violence est le révélateur du courage individuel de chaque militant (c'est parce que la violence s'exerce ou menace de s'exercer que le courage peut se manifester); d'autre part, la violence est la reconnaissance de l'importance de l'organisation en tant que structure d'opposition (si le pouvoir persécute le NBP, c'est que le NBP doit réellement menacer le pouvoir). Pour être effectivement mis en scène - c'est-à-dire pour que la mise en scène soit convaincante - l'héroïsme individuel et collectif des militants du NBP a donc besoin de la répression du pouvoir. Loin d'être une nuisance ou un coût, la répression par les autorités est un ingrédient indispensable à la fierté d'être un natsbol ; comme dans le cas des gangs étudiés par Frederic M. Thrasher ${ }^{38}$, l'existence d'une échelle de valeurs interne, distincte du reste du monde social, explique la recherche paradoxale de la répression par les militants, et en fait une source tout aussi paradoxale de plaisir. Notons enfin, comme le démontrent Combes et Fillieule, que la répression ne fait que renforcer la spécificité de ce code moral de l'organisation, en contribuant à son isolement, " propice à la construction de communautés émotionnelles soudées 39 ».

38. Thrasher F. M., The Gang: a Study of 1313 Gangs in Chicago, Chicago, Chicago University Press, 1963.

39. Combes H., Fillieule O., op. cit., p. 1072. 


\section{La rétribution escapiste et ses conditions}

Les émotions positives générées par la répression, qui constituent l'un des plaisirs du militantisme au NBP, sont malgré tout paradoxales, au sens où elles ne vont pas de soi, apparaissant comme des préférences étranges qui doivent elles-mêmes être expliquées. Pourquoi ces émotions-là, et non d'autres, deviennent-elles source de plaisir pour les militants ? Si elles constituent la partie émergée du fait militant, se matérialisant par des comportements facilement observables, dans les représentations des militants elles n'existent pas séparément d'un cadre de pensée cohérent : celui de l'bistoire que les militants se racontent sur leur activité, ou du jeu auquel ils jouent collectivement. Cette histoire ou ce jeu donnent accès à la définition de la situation par les militants, permettant d'apprécier en quoi consiste l'intérêt spécifique qu'ils retirent de leur engagement.

L'analyse des nombreuses observations de la vie quotidienne des natsbols, ainsi que de leurs témoignages ${ }^{40}$, met en évidence la romantisation dont l'action politique fait l'objet dans leur esprit. Les militants se vivent, en effet, subjectivement comme un groupe de révolutionnaires désespérés (nombreux sont ceux qui, dans les entretiens, se présentent comme « révolutionnaire(s) professionnel(s) : ni maison, ni études, ni travail »); ce faisant, ils mythifient leur présent pour le transformer en poème épique. Dans leur vision des choses, leur adhésion et leur action au NBP s'expliquent, en effet, par l'extrême gravité du contexte politique. C'est parce que la situation du pays est insupportable qu'ils sont là, et en étant là, ils sont insupportables au pouvoir ; il s'agit donc d'un combat sans merci ayant pour but une révolution totale (bien que les raisons du rejet du pouvoir soient extrêmement variables : excès ou au contraire manque de nationalisme ; adhésion ou au contraire mépris des valeurs démocratiques, etc.). Les militants n'envisagent pas ce combat comme de la « politique » (associée à l'ennui, à la routine, à l'impuissance); révolutionnaires, ils font plutôt la "guerre », cette dernière étant pour eux une métaphore à la fois de la situation objective (la répression dont ils font l'objet, les actions d'opposition radicale auxquelles ils se livrent) et de leur état d'esprit (être " en guerre » détermine par exemple l'intensité de la camaraderie dans l'organisation).

La guerre que mènent les natsbols incarne ensuite pour eux une cause perdue, une lutte désespérée. Peu de militants pensent, en effet, que la victoire est probable, voire possible. Le plus souvent, ils se décrivent comme le « mouvement de gens qui n'ont rien à perdre ", des « désespérés " (plusieurs militants utilisent ce qualificatif) : «Notre avenir, c'est la prison ou la mort », affirme ainsi l'un d'entre eux. Pourtant, cet état de désespérance qui devrait, selon la

40. Sauf indication contraire, les citations proviennent des vingt-quatre entretiens avec les militants actifs du NBP. 
logique instrumentale, être un obstacle rédhibitoire à la poursuite de l'action, n'est nullement démobilisant. Il alimente, au contraire, l'image héroïque de soi qu'ont les militants, "soldats de la fin du monde » auxquels le statut de condamnés ne fait qu'ajouter de la grandeur. La lutte des natsbols renferme à leurs yeux une dimension proprement grandiose ; loin de se réduire à son aspect visible, forcément limité (les militants sont peu nombreux, leurs actions peu efficaces), elle est perçue comme « historique ». Unique, le NBP serait la formation politique la plus signifiante de l'époque ; plus encore, le parti transcenderait le cadre habituellement fixé aux organisations politiques à travers sa stature quasi métaphysique.

Cet aspect « historique » du parti en constitue en même temps la dimension romanesque : le NBP s'inscrit dans l'Histoire non seulement en tant que phénomène objectif, mais également en tant que sujet littéraire. En effet, le parti est au centre de plusieurs romans autobiographiques d'Édouard Limonov ${ }^{41}$; aux côtés du leader de l'organisation lui-même, ces écrits mettent en scène des militants dont certains sont devenus des légendes au sein du parti, ainsi que les divers épisodes de l'histoire de l'organisation. Pour beaucoup d'enquêtés, le parti est avant tout un sujet de roman puisqu'ils en apprennent l'existence par les livres de Limonov ; ceux qui n'ont pas lu ces livres avant l'adhésion les lisent ultérieurement, acquérant ainsi le même monde de références. Armés de représentations façonnées par ces écrits où l'organisation est magnifiée par le récit littéraire, les militants vivent ainsi leur engagement comme extraordinaire, au sens où il leur permet d'intégrer le monde de l'histoire mise en roman. La sensation de devenir, en entrant au NBP, des « héros », non seulement au sens d'individus courageux, mais aussi au sens de personnages d'un roman, constitue pour eux l'un des plaisirs les plus intenses. Le mode de vie, le discours, le comportement des militants sont finalement fortement façonnés par leur volonté de correspondre à la description dont ils font l'objet sous la plume de Limonov.

Enfin, le dispositif imaginaire de l'organisation est également alimenté par un ensemble de références historiques à des périodes jugées glorieuses durant lesquelles des individus ou des groupes révolutionnaires sont érigés en exemples. Le moment historique privilégié est le début $\mathrm{du} \mathrm{XX}^{\mathrm{e}}$ siècle ; suivant en cela les écrits des pères fondateurs du parti (Dugin et Limonov) et la ligne éditoriale du journal du parti (Limonka) des années 1990, trois groupes se partagent le haut du pavé : les bolcheviques, les fascistes italiens et les nationalsocialistes allemands. D’autres références (comme par exemple les SR russes) apparaissent également de temps à autre ${ }^{42}$. Ce qui intéresse les militants dans

41. Notamment Anatomiâ geroâ [L'anatomie du béro], Moscou, Rusič, 1997 et Moâ političeskâa biografiâ [Ma biographie politique], Saint-Pétersbourg, Amfora, 2002.

42. Curieusement, les national-bolcheviques - allemands ou russes - ne font pas partie des références les plus utilisées, sans doute parce qu'il s'agit surtout d'individus isolés producteurs de doctrines (comme Ernst Niekisch ou Nikolaï Ustrialov) et non de groupes ayant laissé des patterns de comportement romantique à reproduire. 
chacun de ces groupes, c'est la période où ils n’étaient pas encore au pouvoir : les bolcheviques admirés et imités sont ceux d'avant 1917, membres d'une organisation de révolutionnaires professionnels poursuivis par l'Okhrana tsariste, envoyés en exil en Sibérie ou jetés en prison, et préparant la révolution dans l'ombre. De même, les fascistes et les nazis qu'ils révèrent sont ceux d'avant leur ascension, membres de groupuscules marginaux rêvant d'une prise de pouvoir incertaine, ces comparaisons indiquant en pointillé le futur grandiose de l'organisation. Enfin, à partir de 2005, une nouvelle référence apparaît, liée à l'affaire des "décembristes " : celle précisément aux décembristes historiques - symboles d'idéalisme dans la culture politique russe -, groupe de nobles qui, après une vingtaine d'années d'activité d'opposition clandestine au sein de diverses sociétés secrètes, ont organisé une révolte le 14 décembre 1825 pour contraindre Nicolas I à renoncer au trône, entreprise qui se solde par l'échec et par l'exécution ou l'exil des principaux conspirateurs.

Plutôt que leurs idéologies - disparates, voire mutuellement exclusives -, c'est la radicalité de ces organisations historiques, leur fonctionnement clandestin et la répression (réelle ou fantasmée) qu'elles subissaient de la part du pouvoir en place qui fondent pour les militants l'attrait de ces métaphores historiques filées. Leur point commun est de représenter une source d'excitation romanesque vécue à travers un jeu de rôles qui les intègre dans un scénario cohérent de lutte révolutionnaire, l'ensemble des options de jeu disponibles obéissant à la même règle, énoncée par un militant en entretien : être "ce qui fait que les cheveux se dressent sur la nuque ». La part de la praxis dans cet engagement excède très largement celle de la poïésis, et le "pèlerinage » dans lequel Hirschmann voyait la métaphore de tout militantisme ${ }^{43}$ est ici un pèlerinage dans le royaume enchanté du jeu. C'est l'importance de cette composante « jeu » qui constitue la rétribution principale du militantisme au NBP, et qui parvient à transformer les coûts de l'activité militante - telle la répression - en bénéfices spécifiques.

Il nous reste à préciser les conditions qui favorisent l'émergence d'un tel rapport entre répression et militantisme. Pour qualifier le plaisir que les militants retirent de la mise en jeu du romantisme en politique, nous employons l'expression de rétribution escapiste. Ce néologisme souligne que ce plaisir spécifique n'est lié ni à l'anticipation des réalisations politiques réelles de l'organisation, ni aux bénéfices individuels de type intéressé, mais à la création, au sein de l'organisation, d'une ambiance qui diffère radicalement du monde social routinier perçu comme désenchanté. Cette rétribution est atypique, apparaissant nettement « attentatoire aux règles du jeu 44 » du champ politique ${ }^{45}$. Ce dernier est, en effet, dominé par les règles de rationalité et d'effica-

43. Hirschman A. O., Bonbeur privé, action publique, op. cit., p. 153.

44. Bourdieu P., "La représentation politique : éléments pour une théorie du champ politique ", Actes de la recherche en sciences sociales, $\mathrm{n}^{\circ} 36-37,1981, \mathrm{p} .6$.

45. Comme dans tout champ, les règles y ont, en effet, un caractère contraignant : "C'est un uni- 
cité, et les formes d'action politique romantique en sont historiquement évincées à mesure que progressent l'autonomisation et la rationalisation $\mathrm{du}$ champ ${ }^{46}$. Pour que la «structure [...] “d'offre" de rétribution 47 » d'une organisation politique propose, parmi tous les possibles, une part substantielle de cette rétribution atypique, l'organisation doit donc nécessairement être marginale : tout d'abord parce que cette forme de fonctionnement est exclue $\mathrm{du}$ pôle dominant; ensuite parce que seules les organisations marginales, pauvres en ressources légitimes (financières, sociales, symboliques), ont intérêt à recourir à ce type de rémunération. Ainsi, c'est aux deux extrêmes du champ politique - à l'extrême gauche et à l'extrême droite - que l'on voit se développer des organisations " escapistes », basées sur des scénarios de lutte révolutionnaire ou de société secrète ${ }^{48}$, dont le mode de rémunération des membres peut transformer la répression en principe de plaisir.

Par ailleurs, pour que la répression puisse effectivement rétribuer les militants, ils doivent être sensibles aux scénarios d'action romantique, et tout particulièrement à l'hérö̈sme. Les raisons de cette sensibilité devant être recherchées dans la socialisation qu'ils ont reçue dans leur milieu familial ou scolaire, il est nécessaire de s'intéresser aux caractéristiques et aux trajectoires des militants dont l'engagement est renforcé et non affaibli par la répression ${ }^{49}$. Or, le romantisme, et en particulier sa composante héroïque, tiennent une place particulièrement importante dans la formation littéraire et civique tant en URSS que dans la Russie post-soviétique ${ }^{50}$. Les entretiens biographiques montrent que les militants du NBP, qui appartiennent majoritairement aux petites classes moyennes cultivées, y ont été massivement exposés, intériorisant des dispositions qui favorisent une appréhension sentimentaliste de la réalité sociale.

vers dans lequel sont à l'œuvre des critères d'évaluation qui lui sont propres et qui ne vaudraient pas dans le microcosme voisin. Un univers obéissant à ses propres lois, qui sont différentes des lois du monde social ordinaire. Quelqu'un qui entre en politique, comme quelqu'un qui entre en religion, doit opérer une transformation, une conversion et même si celle-ci ne lui apparaît pas comme telle, même s'il n'en a pas conscience, elle lui est tacitement imposée, la sanction en cas de transgression étant l'échec ou l'exclusion. Il s'agit donc d'une loi spécifique et d'une loi qui est un principe d'évaluation et éventuellement d'exclusion. » (Bourdieu P., Propos sur le champ politique, Lyon, Presses universitaires de Lyon, 2000, p. 52). De même, Bernard Lacroix s'attache à montrer que l'« ordre politique » est un «ordre propre " qui " obéit à des contraintes spécifiques " (Lacroix B., "Ordre politique et ordre social. Objectivisme, objectivation et analyse politique » in Leca J., Grawitz M. (eds.), Traité de science politique, vol. 1, Paris, PUF, 1985, p. 564).

46. Cf. Hobsbawm E., Les Primitifs de la révolte dans l'Europe moderne, Paris, Fayard, 1963, mais aussi Weber M., Le Savant et le Politique, Paris, Plon, 1959 [1919].

47. Gaxie D., op. cit., p. 179.

48. C'est le cas de la deuxième organisation analysée dans notre thèse, l'Union eurasiste de la jeunesse (ESM).

49. Zwerman G., Steinhoff P., op. cit.

50. Ce vaste sujet, qui excède largement les cadres de l'article, est abordé dans notre thèse de doctorat. 
Enfin, tous les contextes ne sont pas également favorables à la vigueur des entreprises escapistes, et donc au rapport affectif à la répression de leurs militants. Ainsi, en temps normal, le champ politique tend non seulement à exclure tout élément hérétique - ce qui marginalise les organisations escapistes - mais à empêcher l'idée même de sa possibilité. Lorsque «l'univers de ce qui est pensable politiquement 51 » est stable, l'émergence d'un rapport romantique à l'action politique, même à la marge, est difficile, tant l'ordre dominant est symboliquement écrasant. En revanche, les moments de crises politiques ${ }^{52}$, et plus généralement, les moments d'instabilité ou de mutation rapide des règles du champ, représentent pour les entreprises hétérodoxes des fenêtres d'opportunité où, parce que tout semble possible, le romantisme est puissamment stimulé. Aussi, le contexte instable et évolutif des années 19902000 - effondrement de l'URSS, crises économiques et sociales qui suivent, arrivée au pouvoir de Vladimir Poutine, révolutions « de couleur » et protestations internes de 2004-2006 - favorise le succès du NBP, et autorise la dynamique répressive positive entre ses militants et le pouvoir.

Le cas du NBP tend à confirmer, si besoin était, qu'aucune relation univoque ne peut être établie entre engagement militant et répression; cette relation est éminemment située et dépend de nombreux facteurs. Ainsi, la position des organisations ou des groupes contestataires dans le champ politique, leur profil en termes de rétributions, le type de références, de théories ou de doctrines qui y sont cultivées, et donc le genre de « jeux » qui y sont joués, les caractéristiques sociales des militants attirés par l'atmosphère qui y règne, mais également la structure globale des opportunités du moment historique, constituent autant d'éléments à prendre en considération lorsqu'on tente d'expliquer une dynamique particulière entre protestation et répression.

Ce cas montre particulièrement que si l'engagement dans des « interactions répressives » avec le pouvoir peut parfois être rentable pour une organisation en termes d'image, de notoriété ou de stratégie, la répression peut également avoir un rendement intérieur non négligeable en nourrissant la rétribution escapiste fondée sur la mise en jeu des scénarios d'action exaltante et anti-routinière. La répression, réelle ou fantasmée, est en effet un ingrédient sinon indispensable, du moins extrêmement payant pour la création et le maintien d'une atmosphère romantique; dans le cas d'entreprises politiques de ce type, il est donc très probable qu'elle alimente l'engagement militant plutôt qu'elle ne le décourage. Comme pour d'autres variantes d'« interactions répressives ", cette configuration paradoxale ne peut être appréhendée qu'en combinant plusieurs niveaux d'analyse : celui des parcours individuels, celui $\mathrm{du}$ fonctionnement des groupes, et enfin celui, beaucoup plus vaste, du

51. Bourdieu P., «La représentation politique : éléments pour une théorie du champ politique ", op. cit., p. 4.

52. Au sens notamment que leur donne Michel Dobry dans Dobry M., Sociologie des crises politiques : la dynamique des mobilisations multisectorielles, Paris, Presses de la FNSP, 1986. 
contexte politique et social dans lequel l'interaction prend place, et qui est plus ou moins susceptible de faire de l'action romantique un attrait. Et si l'amour de la répression reste rare, c'est que les facteurs qui en favorisent l'existence ne sont eux-mêmes que rarement réunis. 